\title{
Effect of mold temperature on short and long-term mechanical properties of PBT
}

\author{
K. Banik* \\ Institute for Composite Materials (Institut für Verbundwerkstoffe GmbH), Technical University of Kaiserslautern, \\ D-67663 Kaiserslautern, Germany
}

Received 11 September 2007; accepted in revised form 29 December 2007

\begin{abstract}
In this work, the effect of mold temperature variation on the short-term mechanical properties obtained from the tensile and Charpy impact tests, and the long-term mechanical properties obtained from dynamic mechanical loading and flexural creep of injection molded polybutylene terepthalate (PBT) are reported. It has been observed that the effect of changing the processing condition viz. mold temperature on the viscoelastic properties are more pronounced when their long-term behavior is tested. The tensile and impact properties showed only a negligible effect to the change in mold temperature. Further, analysis of the creep curves by applying a four-element Burger model presented a comprehensive understanding of their long-term viscoelastic behavior with respect to the change in mold temperature.
\end{abstract}

Keywords: mechanical properties, mold temperature, polybutylene terepthalate, semicrystalline, molecular packing

\section{Introduction}

With the increasing use of PBT in various engineering areas such as electrical, electronic, automotive, industrial and chemical sectors, a critical evaluation of the stress and deformation is needed to predict the reliability and failure behavior of such structures [1-4]. Material property determination is an important aspect of stress analysis. When compared to the conventional materials like steel, wood, etc., polymers exhibit lower strength and modulus. Their viscoelastic behavior reflects the combined effect of the viscous and elastic responses and determines the overall mechanical properties of the finished part. The deformation behavior of polymers is thus quite different from that of the elastic materials. The material response to the loading depends strongly on the timescale over which the perturbing force is applied, temperature and the speed at which they are deformed. This is exhibited in a set of processes like creep, stress relaxation rate or dynamical mechanical behaviour [5-9]. These are generally termed as long-term properties of a material and their testing time is considerably longer than the short-term tensile or impact tests. While accurate, creep has the obvious disadvantage of long test time, often years, and a large number of tests are required to characterize a material. The result is sparse data for polymers that is often difficult to compare and apply to design.

The viscoelastic properties of polymers are to a great extent depending on the thermomechanical history during processing. Generally, the commercial molding processes like injection molding, involve three extreme conditions - very high cooling rates, high pressure and shear and elongational flow while making a polymeric part. Thus the complex thermorheological situations that arise by varying the injection molding process parameters lead to different structural parameters like molecular orientation, residual stress, free volume and 
crystallinity (for a semicrystalline polymer) in the finished part varying along the flow direction as well as along the thickness direction. These can influence the viscoelastic properties of the molding significantly. An extensive review of the effect of processing and more specifically of thermal history on the properties of semicrystalline thermoplastics and its composites has been made by DePorter et al. [10].

This paper presents the results of a study of the effect of mold temperature on the short and longterm properties of injection molded PBT and demonstrates that the process-induced deformation behavior of the injection moldings can be better manifested when their long-term behavior is pursued.

\section{Experimental}

Unreinforced polybutylene terepthalate (Ultradur B 4500) used in this study was kindly supplied by BASF, Germany. Dumbbell-shaped tensile specimens of $4 \mathrm{~mm}$ thickness (Figure 1) were produced using Arburg Allrounder 320S (500-350) injection molding machine equipped with a mold temperature controller at three different mold temperatures, viz. 14,40 and $60^{\circ} \mathrm{C}$ and are henceforth represented by PBT14, PBT40 and PBT60 respectively. All other processing parameters such as injection flow rate $\left(20 \mathrm{~cm}^{3} / \mathrm{s}\right)$, holding pressure $(50 \mathrm{MPa})$ and holding time $(15 \mathrm{~s})$, rest cooling time $(25 \mathrm{~s})$, melt temperature $\left(245^{\circ} \mathrm{C}\right)$ and pressure-to-switch over

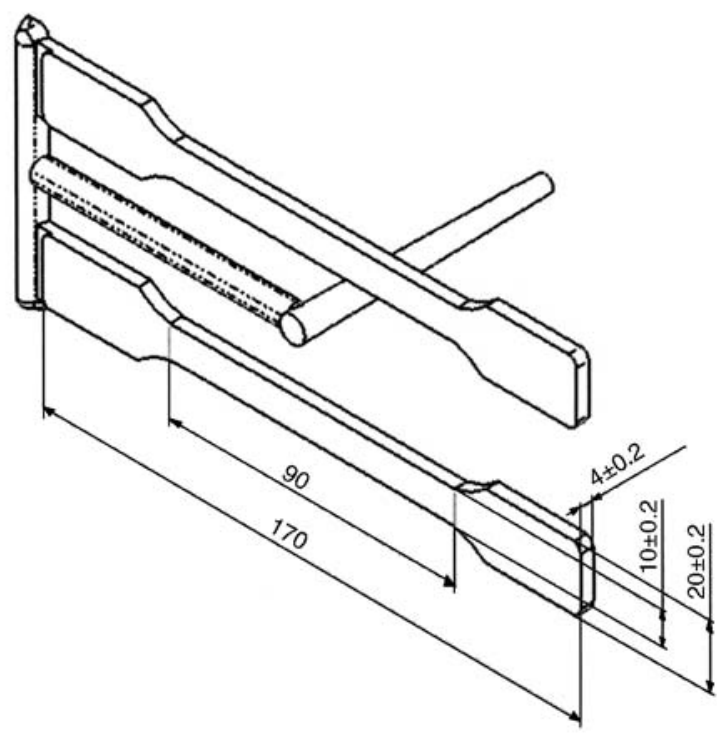

Figure 1. Schematic of the injection molded specimen (all dimensions are in $\mathrm{mm}$ )
(40 MPa) were kept unaltered. Before injection molding, PBT granules were dried at $120^{\circ} \mathrm{C}$ for 3 hours.

Tensile tests were conducted according to DIN EN ISO 527 in a Zwick tensile testing machine. The deformation was measured by laser scanning technique using a cross-head speed of $5 \mathrm{~mm} / \mathrm{min}$. From these short-term tensile tests the characteristic values of tensile strength, elastic modulus and elongation at yield were determined and an average of five test specimens were reported.

Impact tests of the injection molded PBT were conducted in a CEAST impact tester. The notched Charpy impact strength was determined from 5 specimens of each type according to ISO 179.

Dynamic mechanical tests were performed on a DMA Q800 instrument (TA Instruments, USA) in a dual cantilever flexural mode. Specimen with dimensions of approximately $60 \times 10 \times 4 \mathrm{~mm}^{3}$ (length $\times$ width $\times$ thickness) were used and the scannings were performed between $0-200^{\circ} \mathrm{C}$ at a heating rate of $3^{\circ} \mathrm{C} \cdot \mathrm{min}^{-1}$ at $1 \mathrm{~Hz}$.

Flexural creep tests were performed using threepoint bending mode at temperatures ranging from 30 to $80^{\circ} \mathrm{C}$, in the same DMA Q800 apparatus. Isothermal tests were run on the specimens by increasing the temperature stepwise by $10^{\circ} \mathrm{C}$. Prior to the creep measurement, the specimens were equilibrated for $5 \mathrm{~min}$ at each temperature and then the flexural creep behavior was tested for $15 \mathrm{~min}$. All the tests were performed under a constant load of $7 \mathrm{MPa}$, which falls within the linear viscoelastic strain range determined by checking the proportionality condition $[11,12]$. Test specimens of the same dimensions used for dynamic mechanical tests were employed for creep studies and the average of three statistically relevant creep and dynamic mechanical test data has been reported for each kind of specimen.

Differential scanning calorimetry (DSC) was done in a Netzsch 200 DSC instrument (Selb, Germany) to determine the crystallinity of the injection molded specimens. Calibrations were done using tin and all the scans were performed from 20 to $250^{\circ} \mathrm{C}$ at a heating rate of $20 \mathrm{~K} / \mathrm{min}$ by constantly maintaining a nitrogen atmosphere inside the sample chamber. The amount of crystalline phase was determined by taking the ratio of the heat of fusion of the polymer against the heat of fusion for the $100 \%$ crystalline PBT assumed as $145 \mathrm{~J} / \mathrm{g}$ [13]. In 
order to determine the effect of thermomechanical history on the crystallinity content of the injection molded specimens, only the first heating scan was considered during thermal analysis.

The molecular packing (or the free volume) inside the injection molded parts was qualitatively characterized from the density measurements accomplished according to DIN 53479 (procedure A) at ambient temperature in a density measuring instrument provided by Sartorius AG (Goettingen, Germany). Ethanol was used for measuring the density of the samples. Then the specific volume was determined from the relation, specific volume, $v=1 / \rho$ where $\rho$ is the density of the material. There exists a strong theoretical base relating the specific volume of polymers to the free volume available [5, $14,15]$. The advantage of density measurement is that it is comparatively simple and, depending upon the process also very small density variations can be dissolved.

\section{Results and discussion}

Figure 2 shows the DSC thermograms of PBT injection molded at three different mold temperatures. It shows that melting occurs between 227$228^{\circ} \mathrm{C}$ for PBT. An exotherm is observed around $210^{\circ} \mathrm{C}$ which can be identified as recrystallization followed by immediate melting [16]. An additional small exotherm $\left(172-175^{\circ} \mathrm{C}\right)$ is also registered for PBT molded at $14^{\circ} \mathrm{C}$ ascribed to the melting of small and defective crystals. The DSC determined crystallinity was found to be $28.5,30$ and $31.1 \%$ for PBT processed at mold temperatures of 14,40 and $60^{\circ} \mathrm{C}$ respectively. This is expected as during cooling from a higher melt temperature to a lower mold

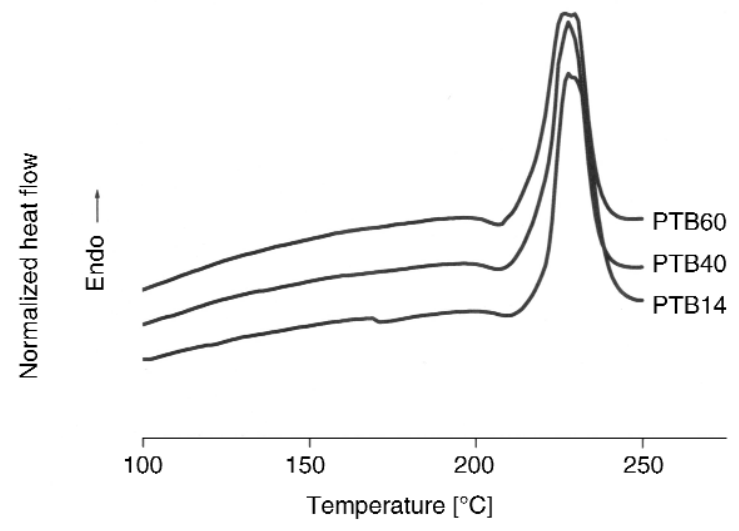

Figure 2. DSC thermograms of PBT injection molded at different mold temperatures

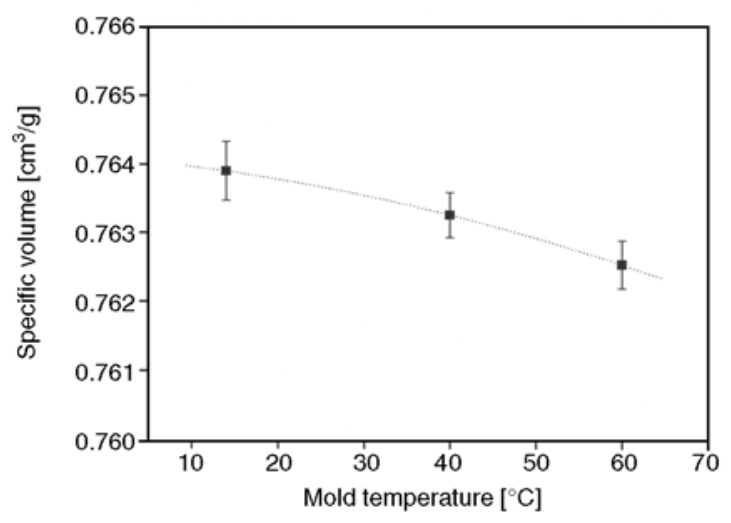

Figure 3. Influence of mold temperature on specific volume of injection molded PBT

temperature, the polymer passes through the crystallization temperature faster for a lower mold temperature than for a higher mold temperature, and so the polymer chains get less time to arrange themselves; contrariwise, slower cooling at higher mold temperature allows the macromolecules to adopt a more regular pattern. So they form bigger crystalline areas resulting in highly crystalline parts. Similar effect was also seen when the density measurements were accomplished. The specific volume was found to increase with the decrease in mold temperature due to the faster cooling at lower mold temperature that freezes-in higher free volume [5]. This has been shown in Figure 3, which clearly suggests higher molecular packing achievable with the PBT specimens processed at higher mold temperatures. Similar results have also been reported in the literature before [17].

Figure 4 shows the results of tensile tests of PBT produced by varying the mold temperature. It should be noted here that the stress-strain diagram of the PBT specimens (not shown here) exhibited a ductile fracture as the material showed yielding

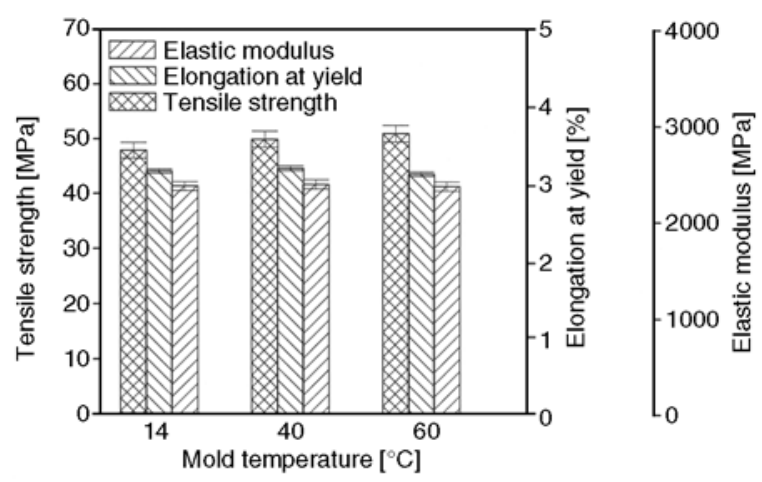

Figure 4. Short-term tensile properties of PBT processed at different mold temperatures 
after which the stress remains fairly constant until it failed. However the results fail to show any significant effect of mold temperature variation on the tensile properties. The elastic modulus increased by only $2 \%$, whereas the elongation at yield showed no remarkable effect when the mold temperature was raised from 14 to $60^{\circ} \mathrm{C}$. Only the tensile strength showed an increase by $8 \%$ on raising the mold temperature. Similar results have also been reported before in the case of amorphous styreneacrylonitrile (SAN) [18]. In the case of amorphous polymers, the deformation behavior depends to a large extent on the free volume or voids present inside the part. But for the semicrystalline polymers like PBT, the deformation process is quite complicated because of the presence of both amorphous and crystalline phases, and the semicrystalline morphology is so complicated that it is sometimes difficult to understand their effect on the deformation behavior as has been seen above. Generally, the short-term tensile tests are very common in practice for determining the mechanical properties of polymeric materials. During tensile testing, a large force acts over the cross-section of the injection molded part for a very short period of time, which is in fact much smaller in magnitude when compared to the average relaxation time of the polymer. So the effect of smaller change in the semicrystalline morphology or the molecular packing on the deformation process is weakly manifested unlike in creep, where the material deforms slowly with time. Hence the influence of mold temperature is little manifested in terms of tensile properties.

Similar effect can be seen from the results of the impact test. This is shown in Figure 5. It shows that

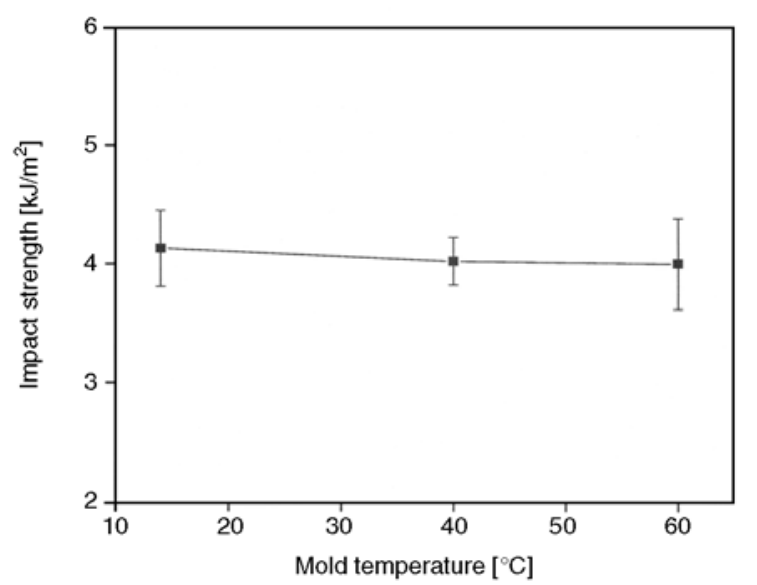

Figure 5. Results of impact test for PBT processed at different mold temperatures with the increase in mold temperature from 14 to $40^{\circ} \mathrm{C}$ there is a little decrease in the impact strength, but no appreciable difference is observed when the mold temperature is changed from 40 to $60^{\circ} \mathrm{C}$. This suggests that the deformation behavior of injection molded PBT is very complicated in the sense that sometimes it does not show any significant effect when the processing conditions are varied. Here we focused some of those results obtained for PBT processed at different mold temperatures. The effect of mold temperature on the dynamic mechanical properties like storage modulus $\left(E^{\prime}\right)$ and loss factor $(\tan \delta)$ is shown in Figure 6. Figure 6a shows the plot of storage modulus as a function of temperature for PBT processed at three different mold temperatures. It shows that with the increase in mold temperature, storage modulus increases as a result of higher molecular packing when the mold temperature is raised. It also indicates that the storage modulus is significantly higher at lower temperatures corresponding to the higher stiffness in the glassy state and decreases with the increase in temperature as the stiffness is greatly reduced once the material passes the glass transition. However at higher temperatures PBT processed at different mold temperatures show similar stiffness. On the other hand, $\tan \delta$ maxima decreased with the increase in the mold temperature (Figure 6b) indicating that the damping of the

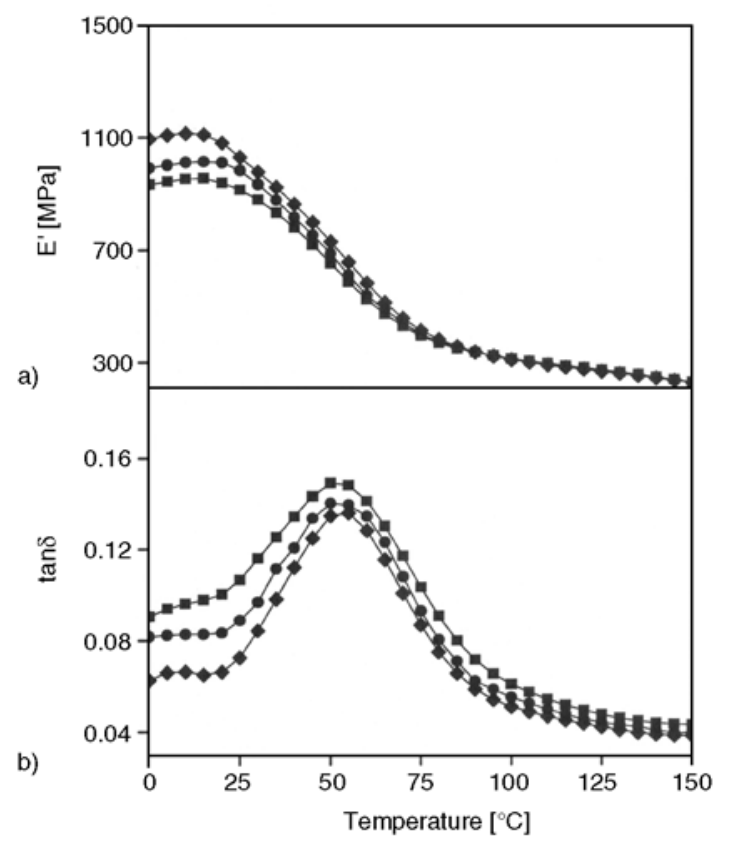

Figure 6. Effect of mold temperature on (a) dynamic storage modulus $\left(E^{\prime}\right)$ and (b) $\tan \delta$ of PBT.

(- PBT14, • PBT40, • PBT60) 
material increases when processed at lower mold temperature. The maximum $\tan \delta$ value decreased by $10 \%$ (from 0.149 to 0.135 ) and the storage modulus at room temperature increased by about $13 \%$ (from 912 to $1028 \mathrm{MPa}$ ) when the mold temperature is increased from 14 to $60^{\circ} \mathrm{C}$. Figure $6 \mathrm{~b}$ further indicates that the glass transition temperature (temperature corresponding to the maxima in the $\tan \delta$ vs. temperature plot) is slightly increased (about $2^{\circ} \mathrm{C}$ ) as the mold temperature is raised. This is anticipated as processing at the higher mold temperature or slower cooling rate can lead to a closer packing of the molecular chains with more crystalline phase and less free volume. Hence more energy is required for the segmental mobility to occur.

The flexural creep behavior of PBT processed at different mold temperatures is shown in Figure 7 where the creep compliance is plotted as a function of time at room temperature $\left(23 \pm 1^{\circ} \mathrm{C}\right)$. The results show higher creep compliance for PBT processed at lower mold temperature indicating that an increase in the mold temperature tends to increase the creep resistance. The creep compliance at the end of the creep test thus showed an increase by about $9 \%$ when the mold temperature is decreased from 60 to $14^{\circ} \mathrm{C}$. This indicates that the strain also increases by $9 \%$ during the creep test due to the decrease in mold temperature, which is hardly observed from the yield strain during tensile test. The difference in creep behavior arises from the difference in the thermal history (cooling rate) that the specimens experience while processing at different mold temperatures. A slower cooling rate at

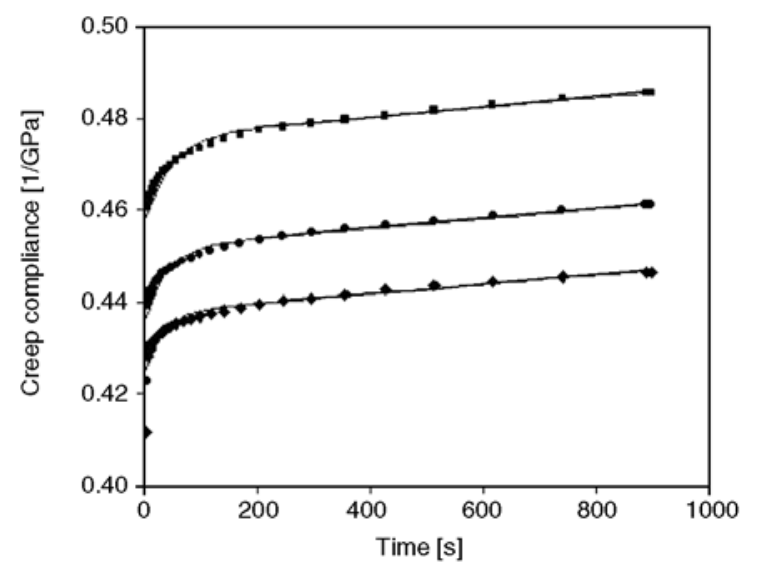

Figure 7. Dependence of creep compliance on the mold temperature of injection molded $\mathrm{PBT}$ at room temperature ( PBT14, • PBT40, • PBT60); solid lines indicate the Burger fit higher mold temperature tends to create more crystalline phase and less free volume that results in densely packed polymer chains and restricts the molecular mobility. This is manifested by lower compliance during the creep test. The observed creep behavior cannot be accomplished to the difference in frozen-in molecular orientation resulting from processing at different mold temperatures [17]. In comparison to the elastic modulus determined from the tensile tests, the variation in creep compliance is quite significant when the mold temperature is changed and can be much pronounced if the creep time is extended. But from the above results it is quite clear that even when the tensile tests do not exhibit any significant difference in terms of short-term mechanical properties on changing the mold temperature, but while considering their long-term behavior, they show a significant effect. Thus it can be suggested that the effect of varying the processing conditions can be better manifested when the long-term behavior of the molded part is tested. On the other hand, the problem of inadequate creep and dynamic data is especially acute for PBT with very little information available in the literature. Since these materials were specifically designed for precision moldings, knowledge of their time-dependent behavior is also essential.

Besides experimental observation, highly developed creep modeling can also be applied to PBT processed at different mold temperatures to develop comprehensive understanding of the creep deformation. In the past half-century, numerous creep models [11, 19-21] have been proposed and applied to describe the creep behavior of viscoelastic materials. Various materials of the same geometry may respond differently under identical external effects. Such differences in response are often attributed to the inherent constitution of the materials. Burger model is a series of combinations of Maxwell and Kelvin-Voigt models (Figure 8) and has been discussed in details in the literatures [22, 23]. According to this model, the total strain is given by the Equations (1) and (2):

$\varepsilon=\varepsilon_{1}+\varepsilon_{2}+\varepsilon_{3}$

$\frac{\varepsilon}{\sigma}=\frac{1}{E_{1}}+\frac{1}{E_{2}}\left[1-\exp \left(-\frac{t E_{2}}{\eta_{2}}\right)\right]+\frac{t}{\eta_{1}}$ 


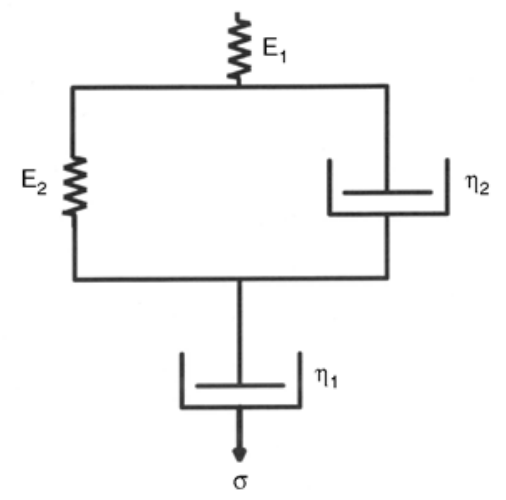

Figure 8. Schematic of the four-element Burger model

where $\varepsilon_{1}$ and $\varepsilon_{2}$ are the elastic and viscous strain represented by the Maxwell model and $\varepsilon_{3}$ is the viscoelastic strain represented by Kelvin-Voigt model, $E_{1}$ and $E_{2}$ are the elastic moduli, $\eta_{1}$ and $\eta_{2}$ represent the viscosity of the material, $\sigma$ is the applied stress and $t$ is the creep time.

The experimental and model plots are shown in Figure 7 for PBT processed at the mold temperatures of 14,40 and $60^{\circ} \mathrm{C}$. The first instantaneous deformation arises from the spring or the elastic element $\left(E_{1}\right)$ and later time dependent deformation comes from the parallel spring and dashpot $\left(\eta_{2}\right)$ and from the viscous dashpot flow $\left(\eta_{1}\right)$. The model parameters $E_{1}, E_{2}, \eta_{1}$ and $\eta_{2}$ can be obtained by non-linear fitting of the experimental data and are shown in Table 1 for the PBT processed at different mold temperatures. According to this model, the modulus $\left(E_{1}\right)$ of the Maxwell spring increases with the increase in the mold temperature, which can be attributed to the stiffening of the material when PBT is processed at higher mold temperature. The retardant elasticity $\left(E_{2}\right)$ however showed a decrease with the increase in mold temperature. On the other hand, viscosity decreased with the decrease in the mold temperature as higher flow occurred in the spring and dashpot and permanent deformation increased. These can be correlated to the microstructure developed in the molded parts, i.e., higher crystallinity as well as molecular packing in the specimens processed at higher mold temperature of $60^{\circ} \mathrm{C}$ is expected to show up with an increase in the

Table 1. Parameters obtained from Burger model

\begin{tabular}{|l|c|c|c|c|}
\hline \multicolumn{1}{|c|}{ Sample } & $\begin{array}{c}\mathbf{E}_{\mathbf{1}} \\
{[\mathbf{G P a}]}\end{array}$ & $\begin{array}{c}\mathbf{E}_{\mathbf{2}} \\
{[\mathbf{G P a}]}\end{array}$ & $\begin{array}{c}\boldsymbol{\eta}_{\mathbf{1}} \\
{[\mathbf{G P a} \cdot \mathbf{m i n}]}\end{array}$ & $\begin{array}{c}\boldsymbol{\eta}_{\mathbf{2}} \\
{[\mathbf{G P a} \cdot \mathbf{m i n}]}\end{array}$ \\
\hline PBT14 & 2.19 & 31.30 & 333.33 & 3.34 \\
\hline PBT40 & 2.30 & 27.73 & 362.32 & 3.64 \\
\hline PBT60 & 2.36 & 25.71 & 438.60 & 3.73 \\
\hline
\end{tabular}

elastic modulus and viscosity that is manifested during the creep test with reduced tendency to creep. Thus the modeling parameters provide a comprehensive understanding of the creep of injection molded PBT and also provide a possible explanation of the internal structure to the viscoelastic property relationship with respect to the change in the mold temperature. Note that the effect of other processing conditions like melt temperature, flow rate and holding pressure on the creep behavior of injection molded PBT have also been studied before, but the results showed that they have only a very little effect on the long-term viscoelastic properties [17] and are hence not discussed in the present study.

\section{Conclusions}

The effect of mold temperature variation on the short and long-term behavior of injection molded PBT were presented. The results indicated that the influence of mold temperature variation is little manifested from the tensile and impact properties of the molded PBT parts, but while considering their creep and dynamic mechanical behavior, they show a significant effect. This deformation behavior could be explained by the influence of processing conditions on the semicrystalline morphology, particularly crystallinity as well as molecular packing density of the molded PBT parts. Moreover, the modeling results provided a comprehensive understanding of the creep behavior. The parameter analysis based on the Burger model provided a possible explanation to the decrease in the creep response on increasing the mold temperature. The long-term properties thus showed more sensitivity to the internal structure than the short-term properties as the characteristic time is of the order of the molecular time. Hence it is suggested that the effect of processing conditions can be better characterized when the long-term mechanical properties of the molded parts are pursued.

\section{Acknowledgements}

A part of this work was conducted in the framework of a research project supported by the German Research Foundation (DFG Me 563/34-1/2). The author is grateful to Prof. Günter Mennig of Chemnitz University of Technology, Germany, for his invaluable suggestions during this study. 


\section{References}

[1] Fakirov S.: Handbook of thermoplastic polyesters. Wiley-VCH, Weinheim (2002).

[2] Shibaya M., Ishihara H., Yamashita K., Yoshihara N., Nonomura C.: Effect of mold temperature on structure and property of PBT injection moldings in the thickness direction. International Polymer Processing, XIX, 303-307 (2004).

[3] Hashemi S.: Temperature dependence of work of fracture parameters in polybutylene terephthalate (PBT). Polymer Engineering and Science, 40, 1435-1446 (2000).

[4] Hashemi S.: Deformation rate dependence of work of fracture parameters in polybutylene terephthalate (PBT). Polymer Engineering and Science, 40, 132138 (2000).

[5] Struik L. C. E.: Physical ageing in amorphous polymers and other materials. Elsevier, Amsterdam (1978).

[6] Crawford R. J.: Plastics engineering. Pergamon Press, Oxford (1987).

[7] Osswald T. A., Menges G.: Materials science of polymers for engineers. Hanser, Munich (1996).

[8] Pegoretti A., Gorigato A., Penati A.: Tensile mechanical response of polyethylene- clay nanocomposites. Express Polymer Letters, 1, 123-131 (2007).

[9] Nagy P., Vas L. M.: Relationship between constant strain rates and stress relaxation behavior of polypropylene. Express Polymer Letters, 1, 84-91 (2007).

[10] DePorter J. K., Baird D. G., Wilkes G. L.: The effects of thermal history on the properties of semicrystalline thermoplastic composites: A review of experimental and numerical investigations. Journal of Macromolecular Science, Part C: Reviews in Macromolecular Chemistry and Physics, 33, 1-79 (1993).

[11] Findley W. N., Lai J. S., Onaran K.: Creep and relaxation of nonlinear viscoelastic materials. North-Holland Publishing Company, Toronto (1976).
[12] Nuñez A. J., Marcovich N. E., Aranguren M. I.: Analysis of the creep behavior of polypropylenewoodflour composites. Polymer Engineering and Science, 44, 1594-1603 (2004).

[13] Moneke M.: Die Kristallisation von verstärkten Thermoplasten während der schnellen Abkühlung und unter Druck. Ph.D Thesis, Technische Universität Darmstadt, Germany (2001).

[14] Struik L. C. E.: Physical aging: Influence on the deformation behavior of amorphous polymers. in 'Failure of Plastics' (eds.: Brostow W., Corneliussen R. D.) Hanser, München 209-234 (1986).

[15] Engelsing K.: Einfluß des freien Volumens auf das verarbeitungsabhängige Deformationsverhalten spritzgegossener amorpher Thermoplaste. Ph.D Thesis, Technische Universität Chemnitz, Germany (2000).

[16] Di Lorenzo M. L., Righetti M. C.: Morphological analysis of poly(butylene terepthalate) spherulites during fusion. Polymer Bulletin, 53, 53-62 (2004).

[17] Banik K., Mennig G.: Process-induced long-term deformation behavior of semicrystalline PBT. Polymer Engineering and Science, 46, 882-888 (2006).

[18] Engelsing K., Mennig G.: Influence of the injection molding process on the free volume and its effect on the creep behavior of styrene-acronitrile copolymer. Mechanics of Time-Dependent Materials, 5, 27-38 (2001).

[19] Ward I. M.: An introduction to the mechanical properties of solid polymers. John Wiley and Sons, West Sussex (1993).

[20] Kolarík J., Pegoretti A.: Non-linear tensile creep of polypropylene: Time-strain superposition and creep prediction. Polymer, 47, 346-356 (2006).

[21] Betten J.: Creep mechanics. Springer, Berlin (2005).

[22] Menard K. P.: Dynamic mechanical analysis. CRC Press, Florida (1991).

[23] Houshyar S., Shanks R. A., Hodzic A.: Tensile creep behaviour of polypropylene fibre reinforced polypropylene composites. Polymer Testing, 24, 257-264 (2005). 